
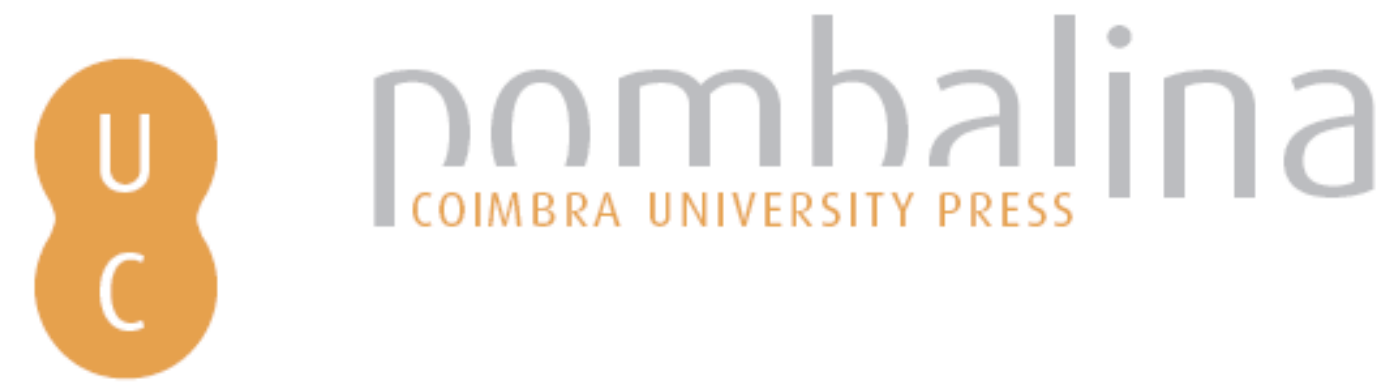

Utilização do GPR para auxiliar na compreensão da evolução da paisagem: estudo de caso do Parque Estadual Veredas do Peruaçu: MG, Brasil

Autor(es): $\quad$ Aranha, P. R. A.; Augustin, C. H. R. R.

Publicado por: Imprensa da Universidade de Coimbra

URL

persistente: URI:http://hdl.handle.net/10316.2/31454

DOI: $\quad$ DOI:http://dx.doi.org/10.14195/978-989-26-0531-9_24

Accessed : $\quad$ 26-Apr-2023 09:59:17

A navegação consulta e descarregamento dos títulos inseridos nas Bibliotecas Digitais UC Digitalis, UC Pombalina e UC Impactum, pressupõem a aceitação plena e sem reservas dos Termos e Condições de Uso destas Bibliotecas Digitais, disponíveis em https://digitalis.uc.pt/pt-pt/termos.

Conforme exposto nos referidos Termos e Condições de Uso, o descarregamento de títulos de acesso restrito requer uma licença válida de autorização devendo o utilizador aceder ao(s) documento(s) a partir de um endereço de IP da instituição detentora da supramencionada licença.

Ao utilizador é apenas permitido o descarregamento para uso pessoal, pelo que o emprego do(s) título(s) descarregado(s) para outro fim, designadamente comercial, carece de autorização do respetivo autor ou editor da obra.

Na medida em que todas as obras da UC Digitalis se encontram protegidas pelo Código do Direito de Autor e Direitos Conexos e demais legislação aplicável, toda a cópia, parcial ou total, deste documento, nos casos em que é legalmente admitida, deverá conter ou fazer-se acompanhar por este aviso.

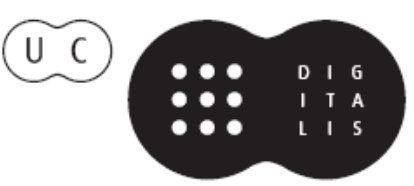





\title{
UTILIZAÇÃO DO GPR PARA AUXILIAR NA COMPREENSÁO DA EVOLUÇÁO DA PAISAGEM: ESTUDO DE CASO DO PARQUE ESTADUAL VEREDAS DO PERUAÇU-MG, BRASIL
}

\author{
THE USE OF THE GPR TO INTERPRETE THE LANDSCAPE \\ EVOLUTION: A CASE STUDY FROM PARQUE ESTADUAL \\ VEREDAS DO PERUAÇU - MG.
}

P. R. A. Aranha ${ }^{1} \&$ C. H. R. R. Augustin ${ }^{2}$

\begin{abstract}
Resumo - Sistema de lagoas localizadas em terrenos arenosos do Supergrupo Urucuia, no Parque Estadual Veredas do Peruaçu, norte do Estado de Minas Gerais, Brasil, são feiçôes comuns nos ecossistemas das veredas, do bioma do Cerrado. A linearidade dessas lagoas sugere que as mesmas possam ter, no passado pertencido a um mesmo sistema, que teria sido deconectado ao longo da evolução da mesma. O objetivo desta pesquisa foi, com o auxílio do GPR e utilizando antenas de 100 $\mathrm{MHz}$ gerar imagens de radargrama que pudessem auxiliar na interpretação das estruturas do fundo de uma das lagoas que formam esse complexo. Resultados desses radargramas indicam uma conformidade muito grande entre a forma abaulada (côncava) do fundo e os sedimentos que alí estão depositados, permitindo assumir que se houve conexão entre a lagoa estudada e as demais localizadas em sua proximidade, isto ocorreu há muito tempo, bem antes da deposição da sequência sedimentar. A espessura $2 \mathrm{~m}$ do pacote sedimentar indica, que ou houve uma sedimentação muito rápida e intensa que pudesse gerar essa espessura, ou ela vem ocorrendo há um tempo geológico considerável.
\end{abstract}

Palavras-chave - sistema laguna, veredas, desconexão, GPR.

Abstract - System of lagoons located in arenaceous lands of Supergrupo Urucuia, in the State Park Veredas do Peruaçu, north of the State of Minas Gerais, Brazil, are common features in ecosystems of veredas, a bioma of the Cerrado (Savannah-Open pasture).

\footnotetext{
${ }^{1}$ Departamento de Geologia, Instituto de Geociências - UFMG; krant_spy@yahoo.com.br

${ }^{2}$ CPMTC - Instituto de Geociências - UFMG; chaugustin@ymail.com
} 
The linearity of these lagoons suggests that the same ones can have, in the past, belonged to the same system, that would have been disconnected throughout the evolution of the lagoon system. The objective of this research is with the help of the GPR and using $100 \mathrm{MHz}$ antennaes to obtain radargram images that could assist in the interpretation of the structures occurring at the bottom of one of these lakes (a dry lake). The results of these radargrames indicate a great conformity between the concave form of the sediments and that of the bottom of the lake, allowing assuming has been occurring since a long time ago. Therefore, if there was a connection between the study lake and those located in its proximity it has occurred a long time, before the deposition of the sequences had been deposited. The thickness of the sediments, $2 \mathrm{~m}$, indicates, that or this deposition was either a very rapid one so that could generate a fast deposition, or it has been taking place during a considerable long geologic time.

Keywords - lagoon system, veredas, disconection, GPR.

\section{1 - Introduçáo}

O Parque Estadual Veredas do Peruaçu, apresenta uma área total de 31.226 ha (IEF 2009) e compreende territorialmente os municípios de Bonito de Minas, Cônego Marino e Januária, no norte do estado de Minas Gerais (Fig. 1). Entre as suas características mais marcantes, destacam-se as veredas, ecossistemas naturais do bioma do Cerrado, identificados pela presença dos Buritis (Mauritia vinera). Em função do seu papel na manutenção dos sistemas hídricos locais e regional, as veredas constituem feiçóes fundamentais que devem ser mantidas e preservadas.

As vertentes do parque, desenvolvidas sobre rochas areníticas dos Grupos Urucuia e Mata da Corda, e extensas áreas no norte de Minas vêem passando por mudanças relativamente rápidas em grande parte decorrentes do aumento da erosão, principalmente as bordas com formas tabulares. Também os seus sistemas hídricos, representados pelas veredas, por áreas úmidas de baixio, por lagoas e rios, apresentam indícios de alteração com processos de diminuiçáo no volume das lagoas e do aumento de sedimentos arenosos nos vales (IEF, 2009).

Isto é especialmente visível no sistema de lagoas próximo à sede do parque, onde foi iniciada a pesquisa sob a coordenação da Dra. Cristina Augustin (2009), com vistas à comprovação da hipótese de que este sistema já tenha integrado parte de uma vereda que, ao longo do tempo, se desconectou.

Para comprovar esta possibilidade foram levantados vários perfis de GPR, de maneira a permitir o acesso ao comportamento e sequenciamento das camadas lenticulares em três lagoas, das quais, uma encontra-se totalmente seca. Devido à sua versatilidade e operacionalidades o GPR vem sendo aplicado a diversas áreas de estudos como a geoctecnia, hidrogeologia, contaminação, estruturação do solo e outros (BENSON, 1995; DAVIS e ANNAN, 1989; HARA et al., 1985; HUGGENBERGER et al., 1994).

Em Minas Gerais, o GPR tem sido utilizado no estudo geomorfológico com sucesso (NEVES et al., 1997; HORN et al., 1998; ARANHA et al., 2002; ARANHA, 2003; AUGUSTIN et al., 2009, Aranha et al., 2009). 


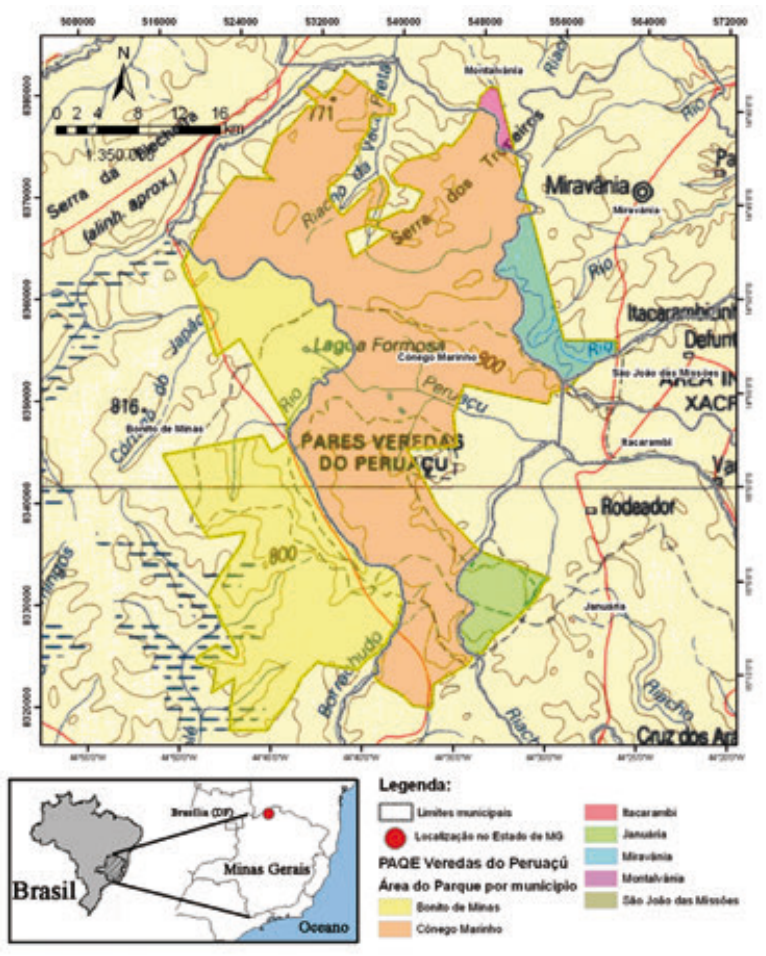

Fig. 1 - Mapa de localização do Parque Veredas do Peruaçu. Fonte: (IEF, 2009).

\section{2 - Características gerais da área}

A área do Parque, localizada entre $15^{0}-16^{0}$ de latitude sul e $45^{0}-46^{0}$ de longitude oeste apresenta relevo de tabular a ondulado, nas altitudes compreendidas entre 650 e $850 \mathrm{~m}$.

Duas coberturas vegetais nativas, são bastante distintas na área: cerrado e florestas. A cobertura vegetal predominante no parque é o Cerrado, do qual faz parte o ecossistema das veredas. Essas ocorrem em pequenas depressóes lineares que formam um sistema complexo de drenagem, no qual se destacam os Rios Peruaçu, Pandeiros e o Cochá, afluentes do Rio São Francisco. Nos afloramentos de calcário observa-se a vegetação que faz lembrar a caatinga, sobretudo pela maior expressão de Cactáceas e Bromeliáceas. Nesses afloramentos ocorre ainda a mata seca, caracterizada pela predominância de espécies totalmente caducifólias nos meses secos.

A classificação climática da área do parque é predominantemente Aw, clima tropical úmido das savanas, que se caracteriza pela existência de estação seca acentuada no período do inverno. A temperatura média anual está em torno de $26^{\circ} \mathrm{C}$, sendo outubro o mês mais quente da região, com $28^{\circ} \mathrm{C}$, e julho o mais frio, com $24^{\circ} \mathrm{C}$, As chuvas distribuem-se de forma irregular e concentrada nos meses de outubro a março (aproximadamente $81 \%$ ), sendo a precipitação média anual de $846 \mathrm{~mm}$ O balanço hídrico apresenta déficit 
entre os meses de abril a setembro, sendo que a umidade relativa apresenta média anual de $72 \%$ (FERREIRA, 1993).

A área amostrada está localizada próxima à sede do parque e faz parte de um sistema de lagoas. Os dados apresentados correspondem aos levantados na Lagoa "Seca" (Fig. 2), localizada a cerca de $200 \mathrm{~m}$ da lagoa da sede do parque, (com água durante todo o ano) e de um perfil obtido após a lagoa cheia, com direção perpendicular ao perfil SECA1.

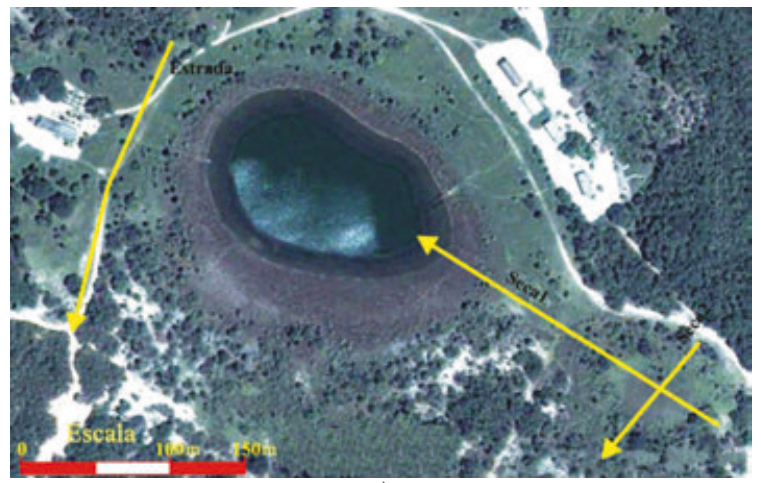

a)

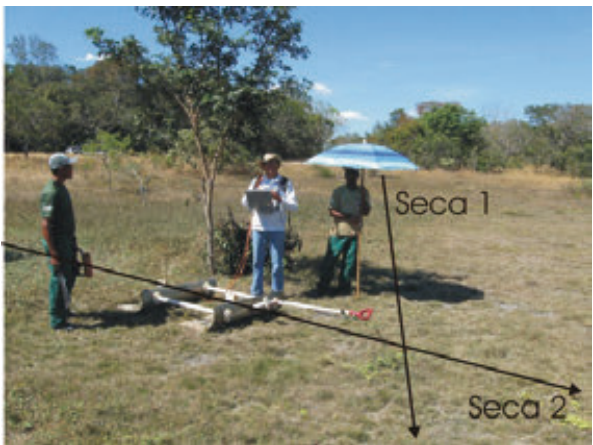

b)

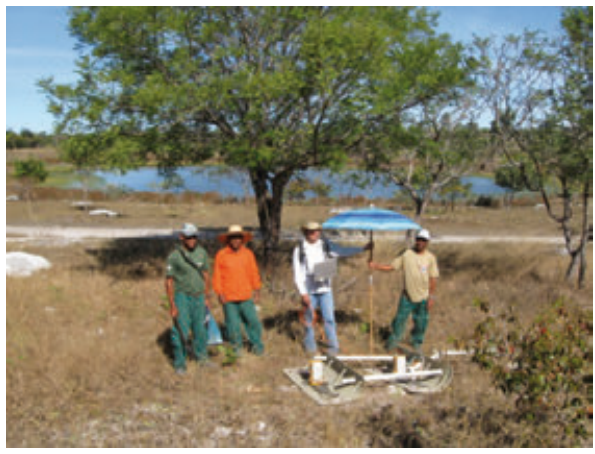

c)

Fig. 2 - Foto da área de aquisição dos dados, a) Imagem com os perfis de GPR obtidos, Seca1, Seca2 e Estrada; b) aquisição dos dados no perfil Secal e Seca2; c) aquisição dos dados no perfil Estrada.

A técnica de uso do GPR é similar, em princípio, à sísmica de reflexão. O GPR produz pulsos eletromagnéticos de altíssima frequência (10 - 3.500 MHz) que são transmitidos para o terreno, através de uma antena emissora. A propagação do sinal depende das propriedades dielétricas do solo onde o sinal (onda eletromagnética) se propagará. As propriedades dielétricas das rochas sáo primariamente controladas pelo seu conteúdo de água. O GPR é sensível às variaçóes de composição ( $\mathrm{pH}$, viscosidade, temperatura, etc.) dos fluidos intersticiais das rochas e às mudanças estruturais destas, preenchidas ou não 
com água. As mudanças nas propriedades dielétricas da rocha causam a reflexão do sinal, que é captado pela antena receptora, e depois na unidade de controle ele é amplificado, digitalizado e gravado.

A campanha geofísica consistiu no levantamento de três perfis de GPR no qual foi utilizado o equipamento RAMAC (Mala GeoScience). A aquisição dos dados foi realizada com antenas de $100 \mathrm{MHz}$.

Os perfis foram obtidos com as antenas em movimento mantendo-se constante a distância entre elas (common offset), no qual o sistema é transportado ao longo de uma direção obtendo-se um perfil das reflexóes versus posição. Foi utilizado o modo de 16 stacks (empilhamento) com o objetivo de aumentar a relação sinal/ruído da onda emitida, melhorando a qualidade dos dados adquiridos no campo.

Os demais parâmetros utilizados na aquisição dos perfis foram: frequência de amostragem de aproximadamente 10 vezes a frequência central da antena utilizada; janela de tempo aproximado de 400ns e espaçamento entre os pontos de aquisiçáo de dados de 0,10 m.

O processamento dos dados objetivou melhorar a qualidade dos resultados obtidos no campo, visando aumentar precisão e obter maior correspondência com a realidade. A edição compreendeu a organização, correção dos dados, o posicionamento das linhas, etc. Manipulaçóes fundamentais foram aplicadas aos dados como decliping, dewow, marcação do "tempo zero", aplicação de ganho AGC, filtragem (filtro gaussiano), correção topográfica e conversão em profundidade, a partir da definição do perfil de velocidade obtido com a interpretação do perfil CMP, registrado no ponto onde os perfis Seca1 e Seca2 se cruzaram. A velocidade encontrada foi de $95 \mathrm{~m} / \mu \mathrm{s}$.

\section{4 - Resultados}

No radargrama do perfil SECA1 (Fig.3) é possível observam-se na parte superior, mais rasa, refletores náo contínuos e intercalados, talvez, devido à presença de materiais mais argilosos intercalados com areias, denotando o sistema de deposição mais recente no fundo da lagoa, agora seca. Logo abaixo, são observados refletores bem delineados, nominados por "A", "B" e "C". Estes apresentam um padrão bem marcado de paralelismo com a topografia na área da Lagoa Seca, entre as distâncias de 0 e $100 \mathrm{~m}$. Logo após, aparece o interflúvio entre as lagoas, seca e cheia.. Após os $160 \mathrm{~m}$, aproxima-se da lagoa cheia. No radargrama esta aproximação é marcada por ondulação dos refletores, e também pela presença de um refletor mais superficial bem marcado. Conforme o observado, nota-se que a estrutura da subsuperfície parece condicionar a presença das lagoas.

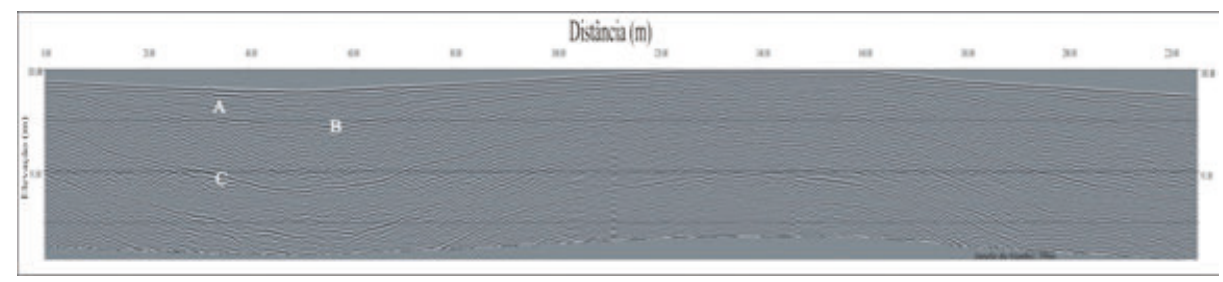

Fig. 3 - Radargrama obtido ao longo do perfil SECA 1 com antenas de $100 \mathrm{MHz}$ (exagero vertical 2:1). 
No radargrama do perfil SECA2 (Fig. 4), perpendicular ao perfil SECA 1, os mesmos refletores "A", "B" e "C" são observados e eles também mantêm o paralelismo com a topografia da superfície. O perfil SECA 1 corta este perfil na distância de $43 \mathrm{~m}$. O perfil SECA 2 corta o perfil SECA 1 na distância de $50 \mathrm{~m}$. Interpretando as duas imagens observa-se que a estrutura da subsuperfície neste ponto forma um disco abaulado.

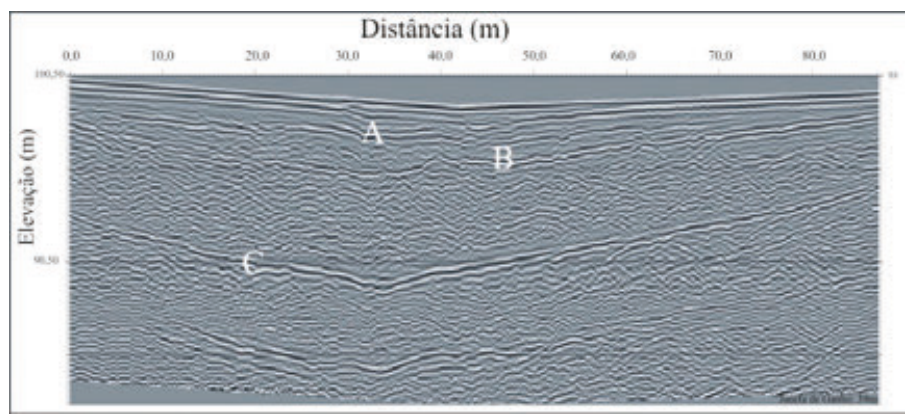

Fig. 4 - Radargrama obtido ao longo do perfil SECA 2 com antenas de 100MHz (exagero vertical 2:1).

O terceiro perfil, chamado de Estrada (Fig. 5), foi obtido logo após a lagoa cheia, no sentido perpendicular ao perfil SECA 1. Neste terceiro perfil aparecem nos primeiros metros, dois refletores bem delineados, que foram nominados "1" e " 2 ". Não foram imediatamente correlacionados com os refletores " $A$ ", "B" ou " $C$ " devido à distância, e também por não apresentarem o mesmo padrão de paralelismo com a superfície, embora o refletor " 1 " pareça apresentar uma ligeira correlação com o formato da topografia. No geral, estes refletores mostram um caimento para a direitano radargrama após a distância de 90m, e entre os dois refletores aparece nitidamente um outro refletor intercalado.

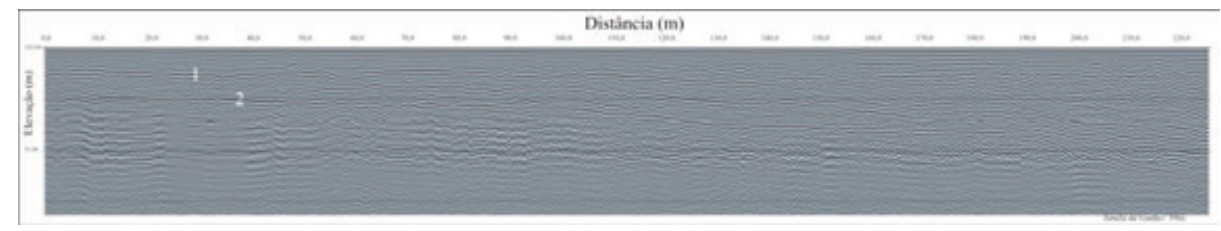

Fig. 5 - Radargrama obtido ao longo do perfil SECA 2 com antenas de $100 \mathrm{MHz}$ (exagero vertical 2:1).

\section{5-Conclusóes}

O sistema lagunar próximo à Sede do Parque Estadual Veredas do Peruaçu, norte do estado de Minas Gerais, é formado por lagoas que contêm água durante todo o ano, e por outras que se encontram secas, mesmo durante os meses chuvosos, de outubro a março. A distribuição espacial das lagoas, tanto as com água, quanto as secas, apresenta simetria que sugere a possibilidade de que as mesmas já tenham estado conectadas. Com a utilização de Ground Penetrating Radar (GPR) foi possivel identificar a estrutura deposicional dos materiais do fundo de uma dessas lagoas (lagoa seca) até $15 \mathrm{~m}$ de profundidade, com 
espessuras variando de 2 até $5 \mathrm{~m}$. Análises dos radargramas mostram que as deformaçóes dos sedimentos acompanham de maneira excepcional, a forma côncava, natural, do fundo da lagoa seca, sugerindo que a deposiçáo tenha ocorrido a relativamente muito tempo. No caso dessas lagoas terem pertencido, no passado, a um mesmo sistema, a desconexão entre dessa lagoa com as demais ocorreu aparentemente antes que esses sedimentos tenham sido completamente depositados. Os dados não são, por si só, suficientes para rejeitar a possibilidade de que essas lagoas jé tenham estado conectadas, indicando a necessidade da realização de pesquisa geofísica, de tradagem e datação do material orgânico com ${ }^{14} \mathrm{C}$, entre outros, para melhor verificação dessa conexão. Contudo, os radargramas foram essenciais para identificar a espessura e a deformação dos materiais sedimentares do fundo desses corpos hídricos, apontando condiçôes de deposição lêntica bem mais antiga do que inicialmente pensado.

Agradecimentos: À Fundação de Amparo à Pesquisa (FAPEMIG) pelo apoio finaceiro (CRA- APQ 0165-09) e ao Instituto Estadual de Florestas-MG (IEF), pelo apoio logístico para a realização desta pesquisa.

\section{Referências Bibliográficas}

ARANHA, P.R.A, AUGUSTIN, C.H.R.R. \& SOBREIRA, F. G. (2002) - The use of GPR for characterizing underground weathered profiles in the sub-humid tropics. Journal of Applied Geophysics, 49(4), 195-210.

ARANHA, P.R.A. (2003). Estudo das coberturas superficiais e sua dinâmica na região de Gouveia, Serra do Espinhaço, MG: utilizando o Radar de Penetração no Solo (GPR). Tese de Doutorado, UFOP, Ouro Preto, 305p.

AUGUSTIN, C.H.R.R. (2009). Análise do escoamento superficial e subsuperficial: entendendo a dinâmica de corpos hídricos em rochas siliciclásticas. Projeto de pesquisa aprovado pela FAPEMIG em 2009 (CRA-APQ 01652-09). 40p.

BENSON, A. K. (1995) - Applications of GPR in assessing some geological hazards: examples of ground water contamination, faults, cavities. Journal of Applied Geophysics, 33, p. 177-193.

DAVIS, J.L. \& ANNAN, A.P. (1989). Applications of ground penetrating radar to mining, groundwater and geotechnical projects: Selected case histories. In: Workshop on Ground Probing Radar, 1988, Ontario: Geological Survey of Canada, paper 90-4, p.49-55.

FERREIRA, E.J. (1993). Análise técnica e econômica do projeto de irrigação do Jaíba, MG. Tese de Doutoramento,Viçosa, UFV, 1993. 159p.

HARA, T. e SAKAYMA, T. (1985). The applicability of ground probing radar to site investigations. OYO, RP- $4159.32 \mathrm{p}$

HORN, A.H; ARANHA, P.R.A., NEVES, S.C., VIANA Fo, L. \& ÁDAMO, R.G. (1998). Novos dados sobre a ocorrência de fosfatos e silicatos de Al-Fe na Serra do Espinhaço Meridional - Perfis nas regiōes da Serra da Miúda e Fazenda Formação - Diamantina, Minas Gerais. In: XL Congresso Brasileiro de Geologia, Seçấo Poster. CD, Belo Horizonte.

HUGGENBERGER, P.; MEIER, E. \& PUGIN, A. (1994). Ground probing radar as a tool for heterogeneity estimation in gravel deposits: advances in data - processing and facies analysis. Journal of Applied Geophysics, V.31, p.171-184.

INSTITUTO ESTADUAL DE FLORESTAS - IEF (2009). Nota Técnica para a Ampliação do Parque Estadual Veredas do Peruaçu. 35p. Acesso em 18.02.2012. 